\title{
Mobility Management for 5G Mobile Networks
}

\author{
Pallavi Sapkale \\ DJSCE \\ Vileparle, Mumbai 400056 \\ India
}

\author{
Uttam Kolekar, PhD \\ APSIT \\ Thane 400615 \\ India
}

\begin{abstract}
The current design and standardization of the next generation or Fifth Generation(5G) will enable new used cases, applications and impressive challenging requirements in terms of mobility performance. For example, next generation mobile networks should support seamless mobility with zero data interruption at each handover, even at high speeds. This work proposes a new research analysis of Mobility Management(MM) solutions in cellular network for next generation. Conventionally Mobility Management solutions were developed for LTE but, from last few years due to increase in demand for high speed seamless mobility without interruption for every handover, the solutions remained incompetent. Hence for improvement in QoS and reducing the delay,this proposed work method will involve the reduction of delay during handoff and analysis for mobility management in both Low speed and High speed scenarios. However, the measurements also reveal that the handover data interruption time can sometimes be hundreds of milliseconds and would try to fulfill the next generation demands. Studies of mobility are conducted for a variety of environments, including generic scenarios with hexagonal network topologies, non uniform site specific scenarios, pedestrian mobility and high speed. So using different network architectures we would implement dual connectivity as well.
\end{abstract}

\section{Keywords}

Cellular network, 5G, Mobility Management(MM), LTE

\section{INTRODUCTION}

Today's life is impossible without networking, rapid growth of android phones is a big challenge for wireless communication. Service providers are attempting to deliver high quality, low latency video and multimedia applications for wireless devices and many more. High speed connectivity is a very basic requirement as we look ahead to next generations of networks[1]. Achieving 24/7 access to, and sharing of, all our "stuff "requires that we continue on our current path: going far beyond simple voice and data services, and moving to a future state of "everything everywhere and always connected ". From last decade, mobile phones have gone through several generations like; 1G, 2G, 3G, 4G and now 5G network is under research [1]. The ongoing development of Fifth Generation mobile communication technology are going to be the cornerstone for applying data and Communication Technology to varied fields, e.g., smart city, smart home, connected automotive, etc.
The Third Generation Partnership Project (3GPP) [2][3], has developed the foremost winning customary technologies within the mobile communication market like Universal Mobile Telecommunication System (UMTS) and future Long Term Evolution (LTE). It is presently polishing off the standardization of each Fifth Generation access network system and Fifth Generation core network system at identical time. In between 3GPP, (SA2) is to blame for identifying the most functions and entities of the future network.

\section{MOBILITY MANAGEMENT FOR LTE}

In next several years, networks is scattered more in nature for such networks, LTE only implements hard handover type. In this process of handovers known as break-before-make handovers, due to hard handover process it will creates major issues in mobility performance for long term evoluation(LTE). So during this process, user equipment is not able to access the data or interchange the data with the help of network. For continuation of data transmission eNB should support because the entity of the Radio Network Controller (RNC) does not exist in LTE[4]. Current mobility management in LTE architectures such as the one employed by LTE, are centralized in nature. To illustrate, the Mobility Management in the LTE architecture shown in Figure 1 which is entrusted with the responsibility of managing mobility of users attached to the network. The given central architecture suffices current day needs. However, due to an exponential growth in traffic and the number of users, these architectures will not be viable for the future $5 \mathrm{G}$ network scenarios. Issues such as scalability,flexibility, blocking and droping will render the current strategies insufficient for the scenarios that will prevail in these future networks [5].

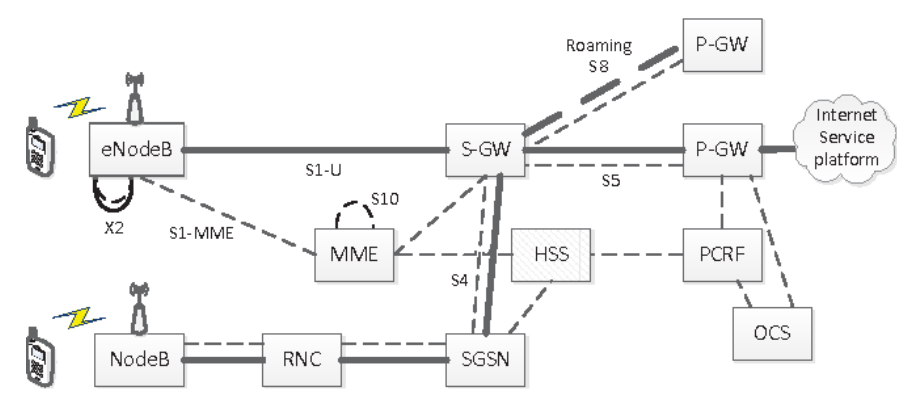

Fig. 1. Architecture for Long Term Evaluation 


\subsection{Mobility Management for LTE-A}

First version of Long Term Evaluation(LTE) came with 3GPP and introduced a mobility enhancements with release 8 which gives more benefit for the UE by increasing the end user throuputs. In heterogeneous networks, UE can simultaneously consume radio sources from macrocell acting as mobility anchor and small cell acting as secondary cell. With these qualities, the UEs benefit from an increased throughput and enhanced mobility robustnesss[6]. Nevertheless, dual connectivity comes with the price of a large number of mobility events. Besides regular handovers, new events are defined for the aggregation, substitution and release of the cells that serve the additional radio links. As will be described in the following parts of this thesis, the increased number of events becomes a challenging issue in high speed HetNet scenarios. Additionally, the selected user plane architecture for implementing DC has an impact on the mobility performance and on the data interruption time perceived by the UEs[7].

\section{MOBILITY MANAGEMENT FOR FIFTH GENERATION OF MOBILE NETWORKS}

As fifth generation $(5 \mathrm{G})$ is expanded and performed, we believe the main dissimilarity collate to $4 \mathrm{G}$ will be the use of much greater spectrum awarding at untapped millimeter wave frequency bands, highly directional beam forming antennas at both the mobile device and base station [8], longer battery life, lower outage probability, much higher bit rates in larger portions of the coverage area, lower infrastructure costs and higher aggregate capacity for many simultaneous users in both licensed and unlicensed spectrum (e.g. the convergence of Wi-Fi and cellular). Figure 2 shows that the Mobil-

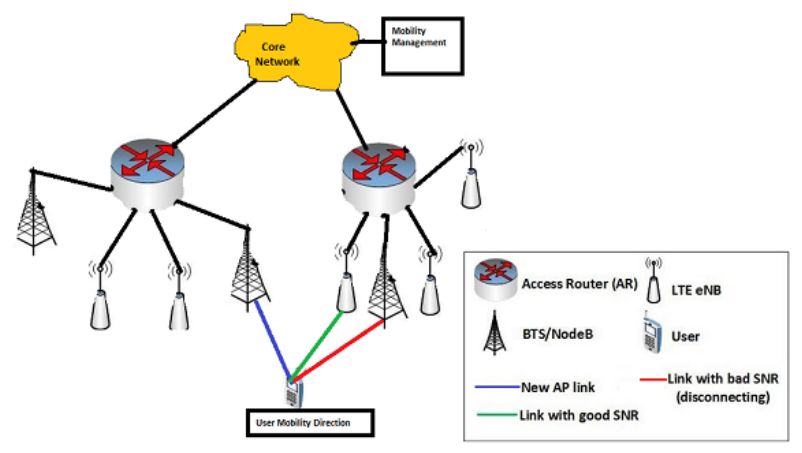

Fig. 2. Mobility Management in 5G

ity Management for 5G[9]. which can be used for the application of SDN Controller. Here services maybe presents on a cloud. Subsequently, parameters from both the user as well as the network can be extracted by the aforementioned MM application. 5G technology has changed to use cell phones within very high bandwidth. $5 \mathrm{G}$ is a packet switched wireless system with wide area coverage and high throughput. 5G technologies use CDMA and millimeter wireless that enables speed greater than $100 \mathrm{Mbps}$ at full mobility and higher than $1 \mathrm{Gbps}$ at low mobility. The $5 \mathrm{G}$ technology provides the mobile phone users more features and efficiency. A user of mobile phone can easily hook their 5G technology gadget with laptops or tablets to acquire broadband internet connectivity[10]. Until now following features of the $5 \mathrm{G}$ technology have come to surface High resolution is offered by $5 \mathrm{G}$ for extreme mobile users. It also offers bidirectional huge bandwidth, higher data rates and the finest Quality of Service (QOS). 5G wireless networks will help 1,000 fold gains in capacity, connections for at least 100 billion devices, and a $10 \mathrm{Gbps}$ individual user experience capable of extremely low latency and response times. Deployment of these networks will emerge between 2020 and 2030. 5G radio access will be built upon both new radio access technologies (RAT) and evolved existing wireless technologies (LTE, HSPA, GSM and Wi-Fi). Success in wireless network innovation will also drive economic and societal growth in exclusively new ways. $5 \mathrm{G}$ will realize networks capable of providing zero distance connectivity between people and connected machines.

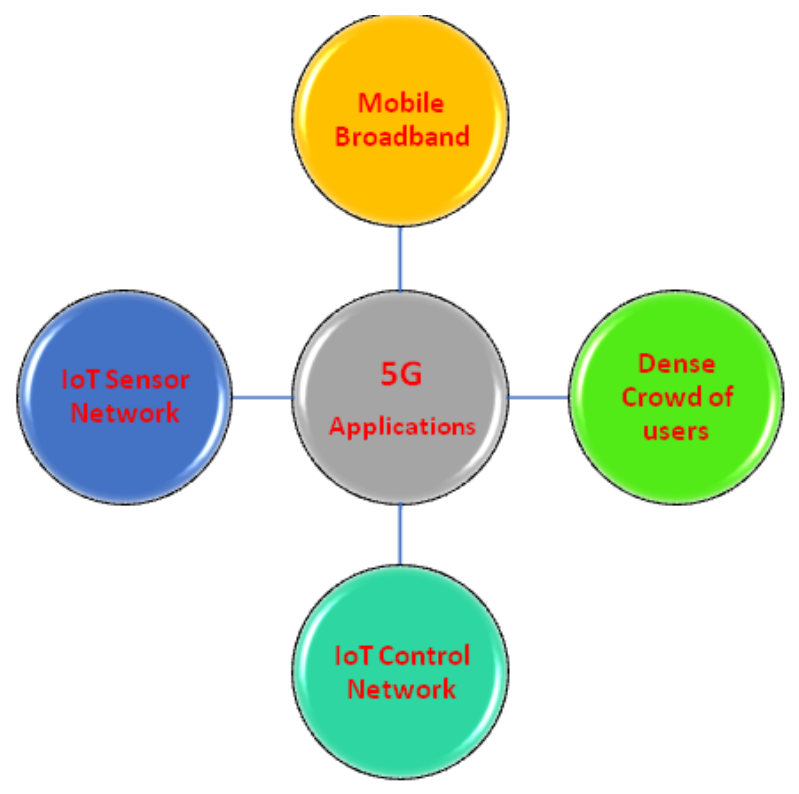

Fig. 3. 5G Applications

Figure 3 5 will be a truly converged system supporting a wide range of applications from mobile voice and multiGigabitpersecond mobile Internet to D2D and V2X (VehicletoX; X stands for either Vehicle (V2V) or Infrastructure (V2I)) communications, as well as native support for Mobile Traffic Control MTC and public safety applications. 3D MIMO will be incorporated at Base Station (BS)'s to further enhance the data rate and the capacity at the macrocell level. Figure 4 shows the architecture of 5G. In which 5G terminal connect the network with General Packet Radio Servics(GPRS) OR Enhanced Data for Global Evolution(EDGE)[11], $3 \mathrm{G}$, WLAN and LTE. And send the data towards different servers, which supports thousands of new applications in various fields.

\subsection{G REQUIREMENTS}

(1) Immersive experience: For virtual real life applications and ultra high defination video requires at least $1 \mathrm{Gbps}$ or more data rates.

(2) Fiber like user experience: $10 \mathrm{Gbps}$ data rates to help mobile cloud service.

(3) Zero latency and response time less than one millisecond latency to support real time mobile control and vehicle-tovehicle applications and communications. 


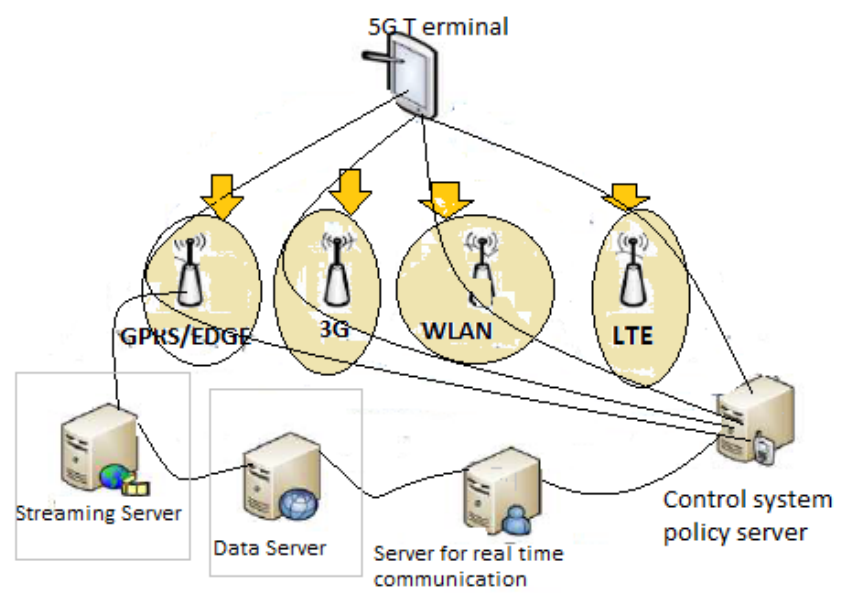

Fig. 4. 5G Architecure

(4) Zero second switching: Maximum 10 millisecond switching time between different radio access technologies to ensure a consistently seamless delivery of services

(5) Massive capacity and always on: Current mobile network systems already support 5 billion users; this will need to expand to also support several billions of applications and hundreds of billions of machines.

(6) Energy consumption: Energy per bit usage should be reduced by a factor of 1,000 to improve the connected device battery life.

\section{ADVANTAGES OF 5G}

(1) High resolution and bi-directional large bandwidth shaping.

(2) Technology to gather all networks on one platform.

(3) More effective and efficient.

(4) Technology to facilitate subscriber supervision tools for the quick action.

(5) Most likely, will provide a huge broadcasting data in Gigabit, which will support more than 60,000 connections.

(6) Easily manageable with the previous generations.

(7) Technological sound to support heterogeneous services including private network.

(8) Possible to provide uniform, uninterrupted, and consistent connectivity across the world.

(9) $5 \mathrm{G}$ technology will encompass spectral bandwidth more than $40 \mathrm{MHz}$ on frequency channel which is a larger range than all other wireless technology systems.

The $5 \mathrm{G}$ new radio (NR) promises a completely new design that will meet more stringent and challenging requirements, allowing the implementation of the envisioned used cases. The design of new $5 \mathrm{G} \mathrm{NR}$ includes the following mobility performance requirements:

- Seamless handovers between cells with zero data interruption time.

- Support for users moving at ultra-high speeds up to $500 \mathrm{~km} / \mathrm{h}$.

-Good mobility performance everywhere.
Table 1. High Speed Scenarios

\begin{tabular}{|l|l|c|c|}
\hline No. & \multicolumn{1}{|c|}{ Parameter } & LTE & $5 \mathrm{G}$ \\
\hline 1 & HO Interuption Time & Intra Freq HO:27.5ms & $0 \mathrm{~ms}$ \\
2 & HO Interuption Time & & $0 \mathrm{~ms}$ \\
\hline 3 & Max UE speed Supported & $350 \mathrm{~km} / \mathrm{h}$ & $500 \mathrm{~km} / \mathrm{h}$ \\
\hline
\end{tabular}

The same good performance should be guaranteed for users in urban scenarios moving at pedestrian speeds and for users in high speed scenarios such us highways or high speed trains.

Above table shows a novel graphical representation[12] of the various functional requirements from the 5G MM mechanisms. The future MM mechanisms are expected to facilitate seamless mobility in the highly heterogeneous 5G networks[13], where multi RAT devices supporting multi connectivity will also be serviced.

\section{SIGNIFICANCE OF THE WORK AND OBJECTIVE:}

- To study and understand concept of MM for existing network , current MM solution for $5 \mathrm{G}$.

- Based on the performance of the existing solutions, will search the critical issues that arise for considering the new mobility requirements.

- Propose new solutions that allows meeting the upcoming mobility requirements and user applications.

- Study and evaluate additional solutions that complement the propose mobility enhancements for meeting the new design specifications.

-Evaluate the suitability of the synchronous handover and the make-before-break techniques for meeting the zero data interruption time at handovers required for the ultra reliable low latency applications. The evaluation must be done by performing a detailed latency analysis of the handover procedures.

—Analysing existing Mobility Management strategies, especially their impacts,

-Developing innovative Mobility Management strategies.

- Based on the previous evaluation, identify the weaknesses and issues of those solutions and propose enhancements for further reducing the interruption time.

-Analyse the possibilities for realizing real make- before-break handovers where the data interruption is eliminated, without increasing the overall handover latency.

- Propose additional mobility enhancements and user association techniques in HetNet scenarios that increase the end user throughput, hence improving the user experience for media content applications.

\section{CONCLUSION}

This paper describes how the foundation for fith generation(5G) Mobility Management(MM) networks can be laid by studying the various researchers for its deployment. This report gives a brief overview of all the changes that would be required in the current scenario for 5G Mobility Management. Considering the various classification of current Mobility Management schemes for 5G and because of its relevance it is very difficult to select a network between User Equipment(UE). So an analysis of the challenges which the design, development and implementation of MM would encounter is also been provided in this paper. Furthermore, the discussions on the challenges also provide insights on the opportunities that exist for future work on mobility management for $5 \mathrm{G}$ network. 
And so, to conclude, MM although faced by multiple challenges will become an important pillar for the future wireless networks, thus enabling them to provide features such as low latency, high data rates, reduced call drops etc., which are primarily also a part of the broader $5 \mathrm{G}$ objectives.

\section{REFERENCES}

[1] Akshay Jain, Elena Lopez-Aguilera and Ilker Demirkol,"Mobility Management as a Service for 5G Networks".

[2] Jahangir Khan, Ali Abbas,Khisro Khan,"Cellular Handover Approaches In 2.5G To 5G Technology"International Journal of Computer Applications (0975 8887), Volume 21 No.2, May 2011

[3] Report ITU-R M.2134.Requirements related to technical performance for IMT- Advanced radio interface(s),2008.

[4] 3GPP Technical report(TR)38.913. 5G; Study on Scenarios and requirements for Next Generation Access Technologies(3GPP TR 38.913 version 14.3.0 Release 14), (2017-10).

[5] Fabio Giust, Luca Cominardi, and Carlos J. Bernardos,"Distributed Mobility Management for Future 5G Networks: Overview and Analysis of Existing Approaches"IEEE Communications Magazine, January 2015.

[6] S.C. Jha, K. Sivanesan,R. Vannithamby,and A.T.Koc, "Dual connectivity in LTE small cell networks",in IEEE Globecom Workshops,Dec 2014,pp 1205-1210.

[7] Athul Prasad, Petteri Lundn, MarttiMoisio, Mikko A. Uusitalo, and ZexianLiwe,"Efficient mobility and traffic management for delay tolerant cloud data in 5G networks",2015 IEEE 26th Annual International Symposium

[8] Gimenez, Lucas Chavarria,"Mobility Management for Cellular Networks: From LTE Towards 5G",Phd thesis, Aalborg University, 2017.

[9] JunseokKima, Dongmyoung Kimb, Sunghyun Choia,“3GPP SA2 architecture and functions for 5G mobile communication system"ScienceDirect ICT Express 3 (2017)18,www.elsevier.com/locate/icte

[10] Lucas Chavarra Gimnez, Simone Barbera, Michele Polignano, Klaus I. Pedersen, Jan Elling, Mads Srensen, "Validation of Mobility Simulations via Measurement Drive Tests in an Operational Network",IEEE 81st Vehicular Technology Conference (VTC Spring), 2015.

[11] D. H. Ring, Cover Sheet for Technical Memoranda. Mobile Telephony Wide Area Coverage. Case 20564, Bell Telephone Laboratories Incorporated, December 1947.

[12] Tom Farley,"Mobile telephone history", Telektronikk 3/4.2005.

[13] A. E. Joel, Mobile communication system, US Patent 3, 663762, 05-16, 1972.

[14] A. Osseiran, J. Monserrat, and P. Marsch, "5G Mobile and Wireless Communications Technology". Cambridge University Press, 2016. 